\title{
Interoperability Criteria and Mechanisms for Seamless Inter-working between UMTS-HSDPA and HIPERLAN/2 Networks Enhanced with MIMO Techniques
}

\author{
Tareq Al-Gizawi ${ }^{1}$, Kostas Peppas ${ }^{1}$, Fotis Lazarakis ${ }^{2}$, Remy Pnitenet ${ }^{3}$, and \\ Jeremy Gosteau ${ }^{3}$
}
${ }^{1}$ Institute of Communications and Computer Science, National Technical University of Athens, 9 Heroon Polytechniou, Zographou, 15773, Athens, Greece tarek@telecom.ntua.gr
${ }^{2}$ Institute of informatics 7 telecommunications, National center of scientific research "Demokritos", Athens, Greece flaz@it. demokritos.gr
${ }^{3}$ Motorola Labs, paris, France, Parc les algorithmes st. Aubin 91193 \{remey.pintenet, jerem.gosteau\} @motorola.com

\begin{abstract}
The concept $4 \mathrm{G}$ wireless communication systems is to provide a user with a rich range of services across different radio access technologies, while maintaining the service's minimum QoS requirement, independently on the coverage area, mobility conditions, and using a single mobile terminal. To achieve this goal the need for interoperable heterogeneous wireless networks emerges. In this article we identify the challenges that arise when enhancing interoperability functionality to two different radio access networks. Specifically we will focus on inter-working UTRA FDD HSDPA and WLANs networks, as two strong candidates for composing the $4 \mathrm{G}$ environment. We also propose an interoperable architecture to achieve seamless inter-working between the aforementioned networks. The article also introduces two interoperable criteria that triggers interoperability based on the use of Cost functions. Namely, these triggers are the initial user assignment to the optimal network and inter-system handover. Each of the two triggers initiates a respective interoperability algorithm. Finally, In order to characterize the interoperability mechanisms behaviour and address an accurate QoS performance analysis, a software simulation platform has been developed. The platform is enhanced with MIMO transceivers and takes into consideration: network configuration, propagation conditions, fast fading, and service requirements.
\end{abstract}

Keywords: Heterogeneous HSDPA, HIPERLAN2, interoperability, Cost function, MIMO, BS, AP

\section{Introduction}

The huge growth of the number of mobile subscribers world-wide, during the last decade, together with the increasing demand of higher transmission rates and flexible 
access to diverse services, motivated significant research standardization and development effort in the area of mobile communication systems. In Europe the transition from $2 \mathrm{G}$ to the $3 \mathrm{G}$ generation has started, and system designers and IST project (ex. FITNESS, METRA, ASILUM) are already working on the 4G technology. The main goals of $4 \mathrm{G}$ networks are to succeed a universal ubiquitous coverage across different radio technologies using a single terminal, and also to provide the $4 \mathrm{G}$ subscriber with rich range of services with variable bandwidth and QoS. These features demand connectivity across multiple networks with different radio technologies, over different geographic areas, as well as for accessing different types of services. This raises the need for interoperable networks.

(4G) communication systems will consist of multi-radio technologies and overlapping wireless networks. The wireless network can be organized in layered structures similar to hierarchical cell structure in cellular mobile radio systems as depicted in fig. 1

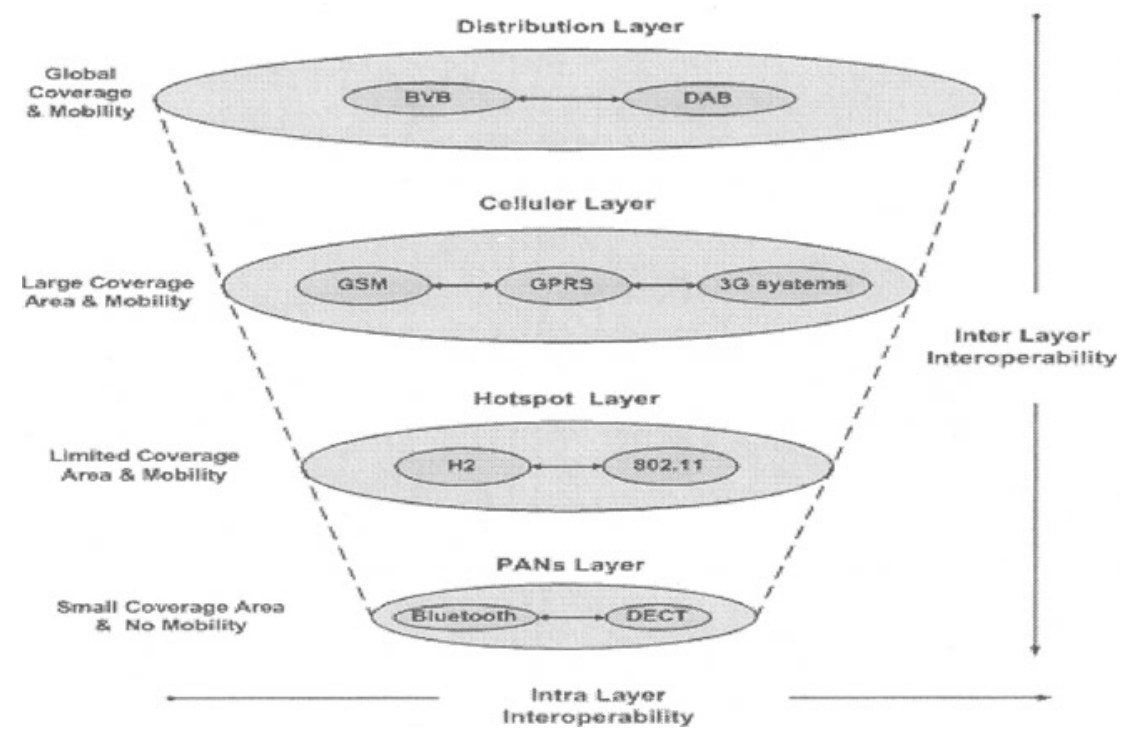

Fig. 1. 4G Layered Architecture

Interoperability can be defined as the capability of a heterogeneous network to support seamless mobility (roaming) between different access radio technologies, maintaining at least the users' minimum QoS requirements.

In this paper we investigate interoperability between High Speed Downlink Packet Access (HSDPA) [1] channel of UTRAN and HIPERLAN/2 [2]. These two radio access technologies can be seen as complementary: the first network supports user mobility while the second supports portability and provides users with higher throughput within limited coverage areas (i.e. hotspots). Introducing a seamless inter- 
working platform would result in higher bandwidth, higher capacity and enhanced QoS. These benefits can be achieved only if inter-network (roaming) enables an efficient management of radio resources. As a result, users can access new services and also select the most appropriate network with an enhanced QoS with respect to the desired service requirements. The achievement of these enhancements depends on the integration architecture of the two technologies and on developing efficient interoperability mechanisms and criteria.

\section{Interoperability Criteria and Mechanisms}

In this section we address the challenges that arise when incorporating interoperability mechanisms and algorithms between HSDPA and WLAN networks. The interoperability platform along with the interoperability criteria and mechanisms that the platform takes into account are also presented.

\subsection{Interoperability Challenges}

When enhancing a $4 \mathrm{G}$ network with interoperability capabilities two main challenges arise at the system level:

- User assignment to the optimal access technology: given that a user may be offered access from more than one wireless technology, one has to consider how the terminal and the network will choose the optimum access technology suitable for the required services. The decision could be taken based on a combination of criteria such as the measured link quality, users' profile, required service and terminal type

- Mobility: mobility is seen as a critical issue when enhancing interoperability in 4G networks. Specifically, at the IP layer the design of mobility enabled IP networking architecture is needed. This includes the exploitation of the existing mobile IP protocols (i.e. Mobile IPv4, Mobile IPv6, and cellular IP $[3,4]$ ) in order to support fast and seamless inter-system handover, QoS and accounting and security issues. A handover at the link layer could trigger a handover procedure at the IP layer in order to minimize IP handover delay. Finally, the strategy and criteria for interoperability at the link level should also be defined. This strategy will depend on the operators' system optimization criterion (i.e. traffic balancing, maximizing the number of satisfied users)

- Re-configurability: the link level algorithms used at the transceiver should be able to adapt to the changes in propagation environment, interference condition, users speed, etc. 


\subsection{Interoperability Platform the $m$ Description (Criteria and Mechanisms)}

The interoperability platform illustrated in Fig. 2 describes our approach to incorporating interoperability between HSPA and WLAN networks. The main concept is to use Cost functions for both the Initial User Assignment (IUA) and InterSystem Handover (ISH) [5]. Each of the two Cost functions monitors the essential parameters at the system level in order to trigger the respective interoperability algorithm. The weights assigned to the Cost functions parameters are tuned adequately. The user computes the relative Cost function. Upon the computed value an interoperability procedure can be triggered. Following the Cost function computation, the target network is also identified. A negotiation between network capabilities and QoS user requirements takes place, leading to a final decision. The weight values of the Cost function are adapted appropriately after a number of simulation runs in order to meet the network optimization criterion for different test cases.

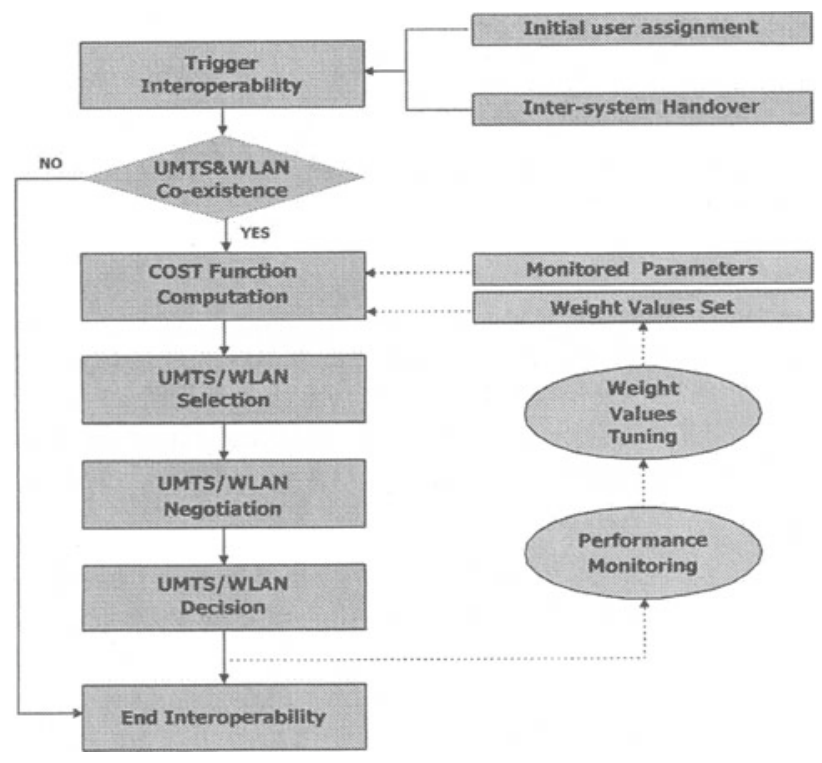

Fig. 2. General Interoperability platform

- IUA Algorithm: When a user is within the joint HSDPA/HIPERLAN/2 deployment the Cost function will be computed to make the decision to which of the two networks the user should initially request access. The parameters of the IUA Cost function are :

- Terminal type: two terminal types (PDAs and laptops) are considered. The weight value assigned to a laptop will contribute in giving higher priority for accessing the WLAN 
- Traffic specification: some services are more adapted to a network due to their characteristics (maximum delay, minimum throughput, priority, security requirements). Hence we take into account the traffic specifications for assigning the service to the appropriate system- e.g. between FTP and web browsing, the former will be given a higher weight to access the WLAN

- Speed: stationary or users moving at walking speed are considered more appropriate to a WLAN, hence they are given a higher weight, which will contribute to giving preference access to the WLAN network

- User preference: depending on the users' preference (profile, given user previous experience, subscription specification or existing connections), a weight will be given in order to contribute to the users' final decision. Users that are not authorized to access either of the two networks may also be considered.

A Cost function can be expressed as a linear combination of the preference functions with the associated weights; hence the IUA Cost function can be expressed as in eq.1 [6]:

$$
C F_{I U A}=\beta_{T T} \cdot P_{f}(T T)+\beta_{T S} \cdot P_{f}(T S)+\beta_{S p} \cdot P_{f}(S p)+\beta_{U P} \cdot P_{f}(U P)
$$

Where:

$P_{f}(X)$ : is the preference function associated to the criterion $\mathrm{X}$,

$\beta_{X}$ are the weights corresponding to the preference function $\mathrm{P}_{\mathrm{r}}(\mathrm{X})$ for the criterion $\mathrm{X}$,

TT: the Terminal Type criterion,

$T S:$ the Traffic Specification criterion

$S p$ : Speed criterion

$U P$ : the User Preference criterion.

The Traffic Preference (TP) ranges from 1 to 4 and is defined as follows:

- TP 1: Requests a WLAN connection and cannot access the HSDPA network

- TP 2: Requests a WLAN connection but can be allocated to the HSDPA network as well

- TP 3: Requests an HSDPA connection but can be allocated to a WLAN network as well

- TP 4: Requests an HSDPA connection and cannot access the WLAN network

The output of the Cost function determines the traffic preference according to the following rule: 


$$
\left\{\begin{array}{lll}
0 \leq C F<0.25 & \Rightarrow & \text { Traffic Preference 1 } \\
0.25 \leq C F<0.5 & \Rightarrow & \text { Traffic Preference 2 } \\
0.5 \leq C F<0.75 & \Rightarrow & \text { Traffic Preference 3 } \\
0.75 \leq C F \leq 1 & \Rightarrow & \text { Traffic Preference 4 }
\end{array}\right.
$$

- ISH Algorithm: The inter-system handover algorithm aims at the successful switching from one network to another, maintaining the minimum QoS requirements for the considered on-going communication.

The proposed ISH Cost function takes into account the following parameters in order to make the decision on when an inter-system handover procedure must be triggered:

- Average Users' throughput: dropping below the minimum QoS requirements based on the average taken over one second of real time simulation.

- Link quality: if the received signal-to-noise ratio results in a Packet Error Rate (PER) that exceeds the upper bound defined by the minimum QoS requirements.

- Mobility: the departure of users from the area where WLAN and HSDPA coexist, and entering an exclusively HSDPA coverage area.

- Traffic load: if the load in one system exceeds a predefined threshold, while the other network has still resources available.

ISH triggering can be categorized as event triggered, periodic, or on demand. When the ISH is triggered, the user computes the Cost function with inputs provided from both user measurements and network signalling information as illustrated in eq. 2:

$$
C F_{I S H}=\beta_{M R} \cdot P_{f}(M R)+\beta_{L Q} \cdot P_{f}(L Q)+\beta_{L o} \cdot P_{f}(L o)+\beta_{\mathrm{Pr}} \cdot P_{f}(\mathrm{Pr})
$$

Where:

$P_{f}(X)$ : is the preference function associated to the criterion $X$, $\beta_{X}$ : are the weights corresponding to the preference function $P_{f}(X)$ for the criterion $X$ $M R$ : the Mobility Requirement criterion

$L Q:$ the Link Quality criterion

Lo: the Load criterion

Pr: the Prediction criterion

If the value of the Cost function computed by the user initiates a handover procedure then: 
- In the case of a WLAN user, a request is made to the HSDPA network. If the contacted Node B cannot satisfy the connection QoS, the user maintains the connection with the current AP but requests repeatedly an access to the Node B until a predefined delay constraint is reached;

- In the case of an HSDPA user, the user requests access to the AP with the best signal-to-noise ratio. If the specific AP cannot satisfy the connection QoS requirements, it tries the AP with the second best received signal-to-noise ratio. The procedure continues until an AP accepts the user or a predefined delay constraint is reached.

In both cases, once the delay constraint is reached, the hand-over request is rejected and the user is dropped.

\section{Scenarios and Simulation Assumptions Considered for the Interoperability Investigation}

In this paper, two interoperability scenarios have been selected for evaluation via a simulation platform enhanced with Multiple Input Multiple Output (MIMO) transceivers.

- The first scenario considers the coexistence of the HSDPA macro deployment with an indoor airport HIPERLAN2 2 hotspot deployment

- The second one represents the coexistence of the HSDPA macro deployment with an outdoor city center HIPERLANI2 hotspot as illustrated in Fig. 2

To investigate interoperability, two common data switched services are considered for both networks, namely; FTP and web browsing with service activity factor of $20 \%$ and $80 \%$ for FTP and web browsing, respectively. Since both networks are enhanced through diversity techniques using multi-antennas, the platform incorporates the appropriate functionalities to evaluate the benefits of MIMO technology, and to examine seamless inter-working between the two networks. The developed simulation platform takes into consideration: network configuration, propagation conditions, fast fading, service requirements and interoperability algorithms. Finally, the full set of simulation parameters can be found in Appendix 1 .

\section{Performance Assessment and Simulation Results}

In order to assess performance of the interoperability algorithms and mechanisms that we have introduced in this paper a system level simulation platform enhanced with MIMO transceivers have been developed. The results were collected from different simulation test cases. Moreover, the performance metric was observed under different traffic loads that correspond to (3, 6, $9 \mathrm{Mbps}$ per Base Station $\backslash$ Access Point). The MIMO enhancement of the simulation platform introduces complexity and high simulation time; hence each simulation run was of 30000 frames (i.e. 1 minute) duration. 
To evaluate the interoperability algorithms we define an appropriate performance metric for both the initial user assignment and the inter-system handover algorithms. Finally, the performance of our algorithms is compared with test cases where the interoperability algorithms were not implemented.
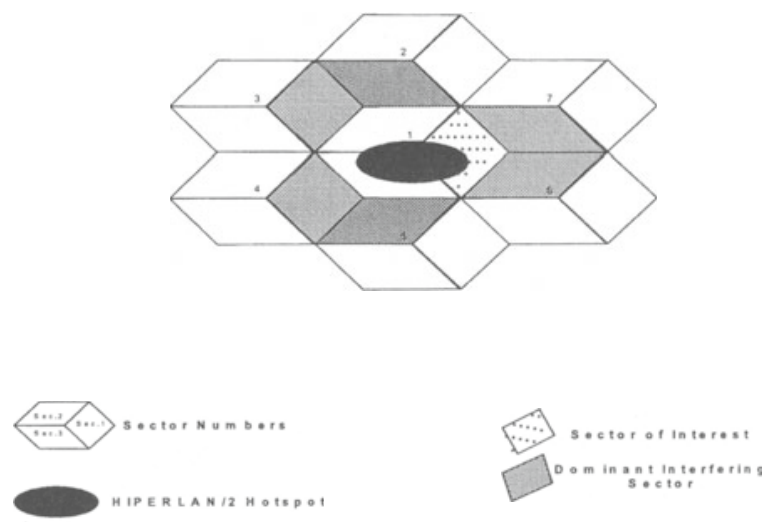

Fig. 3. The Joint HSDPA-HIPERLAN12 Deployment

\section{- (IUA) Simulation Results:}

For the IUA algorithm, the following performance metric is defined:

- Percentage of user preference un-satisfaction (PU_unsat): is the percentage of users that are assigned to a network other than the one of their preference. Since the output of the introduced Cost function may assign a user to a network different from his original preference, $P U_{- \text {unsat }}$ is considered appropriate for performance evaluation.

Table 1 illustrates a comparison between the $P U_{- \text {unsat }}$ in the case where the initial user assignment algorithm is implemented and in the case where the algorithm is not taken into consideration. The results show the significant reduction (about 70\%) of the mean $P U_{- \text {unsat }}$ due to the algorithm implementation. Moreover, the issue of the IUA was found to be independent of the network load. This makes our algorithm robust and applicable to all traffic conditions

Table 1. PU_unsat Vs. Algorithm application

\begin{tabular}{|c|c|c|}
\hline & $\begin{array}{c}\text { With IUA } \\
\text { algorithm }\end{array}$ & With no IUA \\
\hline$P U_{\text {_msat }}$ & $19.7 \%$ & $64.6 \%$ \\
\hline
\end{tabular}

In Fig. 4, we illustrate the results of a test case where the $P U_{- \text {unsat }}$ was computed for all possible combinations of weight distributions of the four parameters of the Cost function (with weight step=0.05). Each one of the points $(a, b, c, \ldots)$ on the horizontal axis represent a weight combination that corresponds to a $P U_{- \text {unsan }}$ value. These weight combinations are adressed in Table 2. For example, we can see that point (b) corresponds to the case where equal weights of 0.25 are assigned to all of the four parameters of the Cost function and results in a $P U_{- \text {unsat }}=27.5 \%$. Based on Figure 5, 
service providers can determine the desired $P U$ the respective weights to the four parameters.

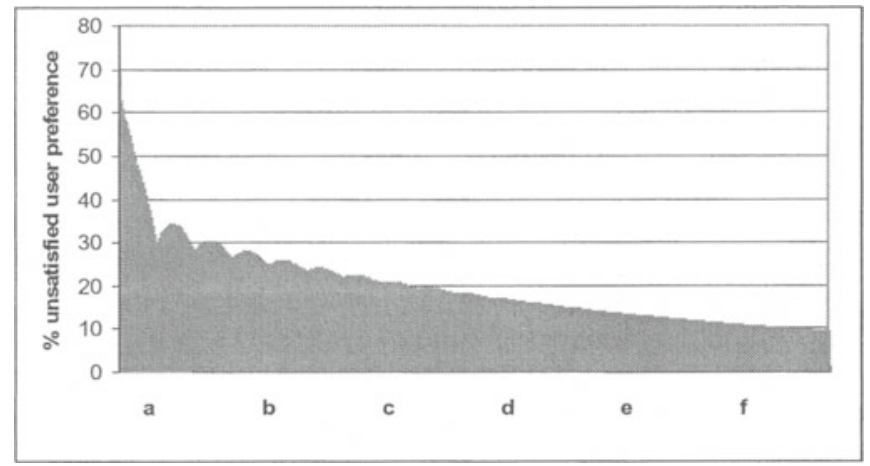

Fig. 4. PU_unsat Vs. Different weight contribution of the Cost function parametres

Table 2. Different combination of wieght values assigned to the Cost function parameters

\begin{tabular}{|c|c|c|c|c|}
\hline & $\begin{array}{c}\text { Terminal } \\
\text { Type }\end{array}$ & $\begin{array}{c}\text { Traffic } \\
\text { Specification }\end{array}$ & Speed & $\begin{array}{c}\text { User } \\
\text { Preference }\end{array}$ \\
\cline { 2 - 5 } & W1 & W2 & W3 & W4 \\
\hline a & 0.1 & 0.85 & 0.65 & 0.65 \\
\hline b & 0.25 & 0.25 & 0.25 & 0.25 \\
\hline c & 0.45 & 0.15 & 0.8 & 0.75 \\
\hline d & 0.6 & 0.5 & 0.4 & 0.4 \\
\hline e & 0.75 & 0.5 & 0.55 & 0.45 \\
\hline f & 0.9 & 0.75 & 0.75 & 0.85 \\
\hline
\end{tabular}

Fig. 5 investigates the effect of each of the four parameters separately on the $P U_{- \text {unsat }}$. In this test case, the $P U_{- \text {unsat }}$ was calculated for all possible weight values assigned to the parameter of interest, while the remaining three parameters were considered to have a weight equal to 0.1 . We can see that all parameters follow similar behaviour; assigning higher weights results in a smaller $P U$

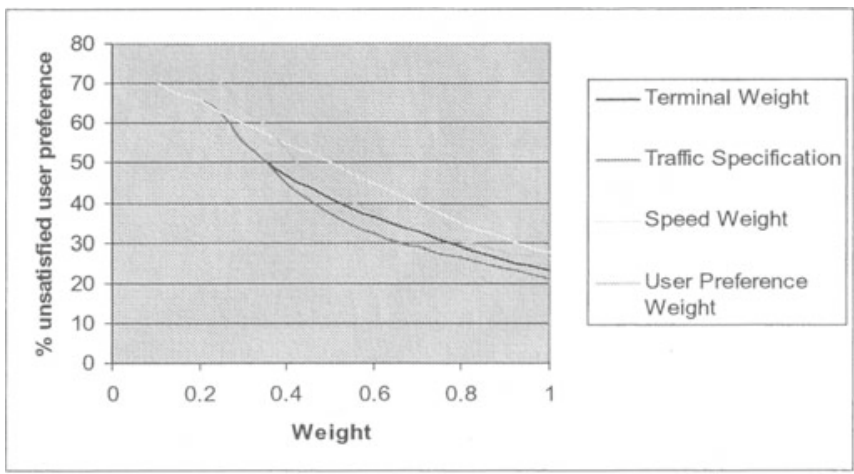

Fig. 5. Cost function parametres Vs. PU_unsat 


\section{- ISH Simulation Results}

For the ISH algorithm, the following performance metric is identified:

- The Handover Drop Rate is identified as the appropriate performance metric for evaluating the inter-system handover algorithm. The Handover Drop Rate is defined as the percentage of dropped handovers as given below

Where:

$$
\text { Drop_rate }=\frac{N_{\text {HO_dropped }}}{N_{\text {HO_tot }}}
$$

- $\mathrm{N}_{\text {Ho_droped }}$ is the number of dropped handovers in the system (both networks)

- $\mathrm{N}_{\text {Ho_tot }}$ is the total number of handovers that occurred in the system

Fig. 6 shows the total number of intra and inter-system handovers that were observed during a simulation run for four different network loads. Since higher network loads correspond to a larger number of users within the deployment, the increase of the number of handovers is considered reasonable.

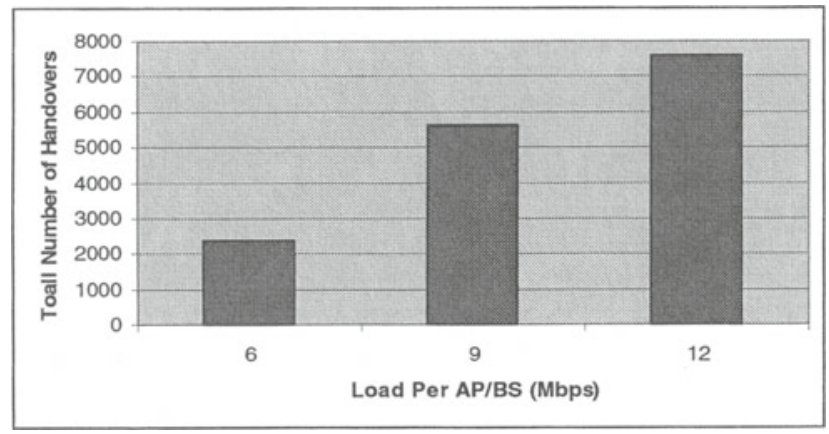

Fig. 6. Network Load Vs. Total number of handovers

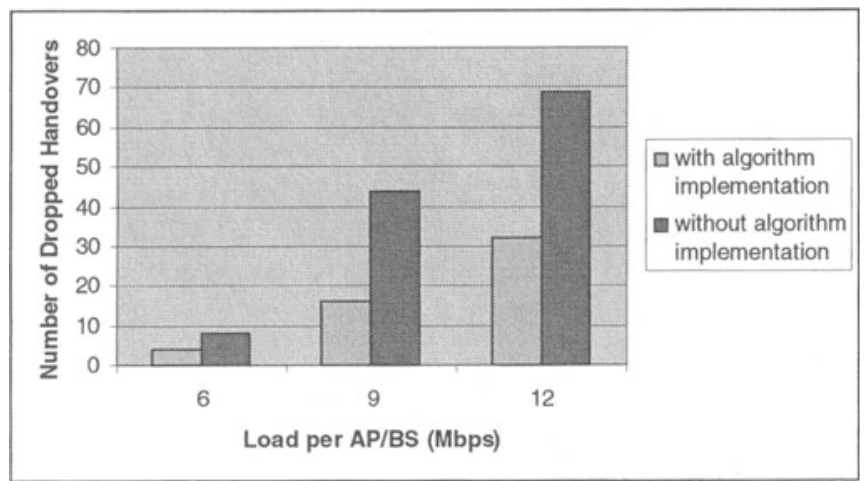

Fig. 7. Network Load vs. Number of dropped handovers

Finally, Fig. 7 shows the performance enhancement achieved by means of the intersystem handover algorithm. The test case compares the number of dropped handovers when inter-system handover is incorporated within the simulation platform vs. the case where the system supports only intra-system handover. The implementation of 
the algorithm achieves approximately $60 \%$ reduction in the handover drop rate for low traffic loads $(3,6) \mathrm{Mbps} \mathrm{BS} / \mathrm{AP}$ and $40 \%$ reduction in high traffic load test cases (9) Mbps BS/AP.

\section{Conclusions}

This paper has proposed an approach to enable inter-operability between HSDPA and WLAN by introducing initial user assignment and inter-system handover mechanisms. Realistic scenarios have been developed to demonstrate the efficiency of the proposed mechanisms and criteria through system simulations. Performance characterization of the two inter-operability mechanisms was also presented and compared with the case where the algorithms were not implemented. The efficiency of the algorithms was proved since the initial user assignment algorithm distributes the users in both networks in a way that reduces the $P U_{- \text {unsat }}$ from $64.6 \%$ to $19.7 \%$ while the inter-system handover algorithm achieves approximately $60 \%$ reduction in the handover drop rate for low traffic loads $(3,6) \mathrm{Mbps} \mathrm{BS} / \mathrm{AP}$ and $40 \%$ reduction in high traffic load test cases (9) Mbps BS/AP.

Acknowledgments. The authors wish to thank all contributors of the IST- FITNESS project within which these studies have been conducted. Deliverables are available on the project web site

\section{References}

1. 3GPP TR 22.934 V6.1.0 (2002-12): Feasibility study on 3GPP system to Wireless Local Area Network (WLAN) inter-working (Release 6)

2. ETSI/TR 101683 V1.1.1 (2002-02): BRAN, HiperLAN Type 2, System Overview

3. http://www.ctr.columbia.edu/ andras/cellularip

4. Ramjee et al., "HAWAII: A Domain -based Approach for Supporting Mobility in Wide-area Wireless Networks", International Conference on Network Protocols, ICNP'99, June 1999

5. R.Pintenet, J.Gosteau, T. Al-Gizawi, F.Lazarakis, K. Peppas, A. Alexiou, VTC fall 2003, “ Evaluation of interoperability mechanisms for Co-existing HSDPA and WLAN enhanced with MTMR techniques

6. http://www.ist-fitness.org 